\title{
Влияние акцепторной примеси бериллия на оптические свойства монокристаллического AIN
}

\author{
(C) Е.Н. Мохов ${ }^{1}$, М.К. Рабчинский ${ }^{1}$, С.С. Нагалюк ${ }^{1}$, М.Р. Гафруров ${ }^{2}$, О.П. Казарова ${ }^{1}$ \\ ${ }^{1}$ Физико-технический институт им. А.Ф. Иоффе Российской академии наук, \\ 194021 Санкт-Петербург, Россия \\ ${ }^{2}$ Казанский (Приволжский) федеральный университет, \\ 420008 Казань, Россия \\ E-mail: Mokhov@mail.ioffe.ru
}

Поступила в Редакцию 23 октября 2019 г.

В окончательной редакции 29 октября 2019 г.

Принята к публикации 29 октября 2019 г.

\begin{abstract}
Исследовано влияние высокотемпературной $\left(T=1880^{\circ} \mathrm{C}\right)$ диффузии ионов бериллия на свойства монокристаллического нитрида алюминия. Показано, что постростовое легирование AlN бериллием приводит к компенсации мелких донорных центров кремния, входящих в решетку AlN неконтролируемым образом в ходе роста. Установлено, что введение Be в решетку AlN приводит к снижению оптического поглощения последнего в видимом и ультрафиолетовом диапазонах. Совокупность результатов объясняется сдвигом положения уровня Ферми, вызванным введением акцепторной примеси бериллия, в сторону потолка валентной зоны AlN.
\end{abstract}

Ключевые слова: AlN, примесь Be, диффузия, оптические свойства.

DOI: 10.21883/FTP.2020.03.49022.9295

\section{1. Введение}

Нитрид алюминия (AIN) является полупроводником с экстремально широкой запрещенной зоной $\left(E_{g}=6.0\right.$ эВ) [1]. Это свойство, в совокупности с развитой технологией получения объемных монокристаллов [2], делает AlN идеальной подложкой для роста светоизлучающих структур на тройных соединениях тринитридов (InGaN, AlGaN) [3]. Для эффективного использования подложки необходимо минимизировать оптическое поглощение (ОП) на рабочих длинах волн предполагаемого светоизлучающего устройства. Относительно недавно были продемонстрированы излучающие в UV-C диапазоне (210 нм) $p-i-n$-диодные структуры на основе эпитаксиальных слоев $\mathrm{AlN}$, легированных $\mathrm{Si}$ (донор) и $\mathrm{Mg}$ (акцептор) [4]. Чтобы сделать такие устройства более эффективными, требуются слои AIN с разумными концентрациями свободных электронов и дырок. Основные свойства донорных примесей $(\mathrm{Si}$ и O изучены довольно полно [5-7], тогда как свойства акцепторных примесей первой и второй групп, Be и $\mathrm{Li}$, и их влияние на оптические и полупроводниковые характеристики AIN мало изучены. Тем не менее недавние исследования монокристаллического AlN, легированного Ве посредством высокотемпературной диффузии из паровой фазы, показали, что Ве является быстро диффундирующей примесью, формирующей глубокие уровни акцепторного типа [8]. Последнее может быть использовано с целью компенсации неконтролируемых примесей $n$-типа, легируюших AIN в ходе роста, таких как кислород и кремний [7], что необходимо для получения монокристаллов AlN с экстремально высоким удельным сопротивлением. Эффект компенсации при- месных центров также может приводить к тушению полос оптического поглощения [8], что необходимо для использования AlN в оптоэлектронных устройствах.

В данной работе нами были исследованы свойства монокристаллов AlN, выращенных методом высокотемпературной сублимации из паровой фазы (PVT) и легированных посредством высокотемпературной диффузии из паровой фазы примесью бериллия, методами электронного парамагнитного резонанса (ЭПР), оптического поглощения (ОП) и спектроскопии комбинационного рассеяния света (КРС).

\section{2. Экспериментальная часть}

В настоящей работе исследовались кристаллы AlN, выращенные методом сублимации (Physical Vapor Transport (PVT)) [9]. Вначале выращивались затравки $\mathrm{AlN}$ на подложках $\mathrm{SiC}$. Затем подложка $\mathrm{SiC}$ сошлифовывалась, и процесс роста AlN продолжался в установках, обеспечивающих получение монокристаллов высокого структурного совершенства с низким содержанием примесей $\left(<10^{19} \mathrm{~cm}^{-3}\right)[2]$.

После выращивания слиток AlN разрезался на пластины, поверхность которых шлифовалась и полировалась. Примесь бериллия (Be) вводилась в кристалл $\mathrm{AlN}$ путем высокотемпературной диффузии. Диффузия Ве осуществлялась из паровой фазы в замкнутом контейнере, сделанном из карбида тантала (ТaC).

В качестве источника диффузии использовался металлический Ве. Температура диффузии Be в AlN варьировалась в диапазоне $1800-2050^{\circ} \mathrm{C}$, а продолжительность процесса составляла 0.5-8 ч. Образцы AlN помещались 
в зону с минимальным перепадом температуры, чтобы минимизировать массоперенос в процессе диффузии. В результате в процессе диффузии не наблюдалось заметного изменения толщины и веса образца.

После проведения диффузии изменялся цвет образца от первоначально янтарного до практически прозрачного [8]. Спектр высокотемпературной люминесценции в видимой области спектра смещался в коротковолновую область. По толщине слоя с измененным цветом люминесценции можно было оценить коэффициент диффузии $\mathrm{Be}$ в $\mathrm{AlN}$, который находится в пределах $10^{-8}-10^{-7} \mathrm{~cm}^{2} / \mathrm{c}$. Таким образом, Ве является быстро диффундирующей примесью в $\mathrm{AlN}$, так же как и в $\mathrm{SiC}[10]$.

Исследование методом ЭПР проводилось с использованием спектрометра $X$-диапазона $(\approx 9.3$ ГГц) Bruker Elexsys E600 в непрерывном режиме. Спектры оптического поглощения регистрировались с использованием спектрометра Shimadzu-2450 UV/Vis. Структурное coвершенство кристаллов до и после диффузии подтверждалось изучением спектров комбинационного рассеяния света, полученных на спектрометре, оснащенном конфокальным микроскопом. Для возбуждения спектров КРС использовался $\mathrm{Nd}$ : YAG-лазер с $\lambda=532 \mathrm{Hм}$ (2.33 эВ). Спектры КРС были получены в геометрии обратного рассеяния $z(x x) \bar{z}$. Чтобы отделить влияние диффузии Be на $\mathrm{AlN}$ от возможных процессов, индуцированных в кристаллах $\mathrm{AlN}$ за счет высокотемпературного нагрева, были исследованы кристаллы, подверженные отжигу при температуре диффузии $\left(1880^{\circ} \mathrm{C}\right)$. Таким образом, измерения характеристик кристаллов AlN проводились до процедуры высокотемпературной диффузии, после высокотемпературного отжига и после диффузии.

\section{3. Результаты и обсуждение}

На рис. 1 представлены спектры ЭПР, зарегистрированные при температуре $T=300 \mathrm{~K}$ в монокристалле AlN до и после процесса диффузии. Сигнал ЭПР, характеризуемый $g$-фактором $g=1.990$, соответствует примеси кремния, представляющей собой мелкий донорный центр в нейтральном зарядовом состоянии $d^{0}$ (электронный спин $S=1 / 2$ ) в позиции замещения в подрешетке $\mathrm{Al}\left(\mathrm{Si}_{\mathrm{Al}}\right)$ [7]. Данное наблюдение свидетельствует о том, что уровень Ферми расположен вблизи дна зоны проводимости и исходный монокристалл имеет n-тип проводимости.

Стоит отметить, что длительный отжиг кристалла при температуре, равной температуре диффузии, не привел к значительным изменениям в спектре ЭПР, как это видно на рис. 1. После проведения процедуры высокотемпературной диффузии ионов бериллия из паровой фазы в кристаллическую решетку AlN спектр ЭПР значительно изменился, а именно пропал сигнал ЭПР, связанный с мелкими донорами $\mathrm{Si}_{\mathrm{Al}}$, как это видно на рис. 1. Для

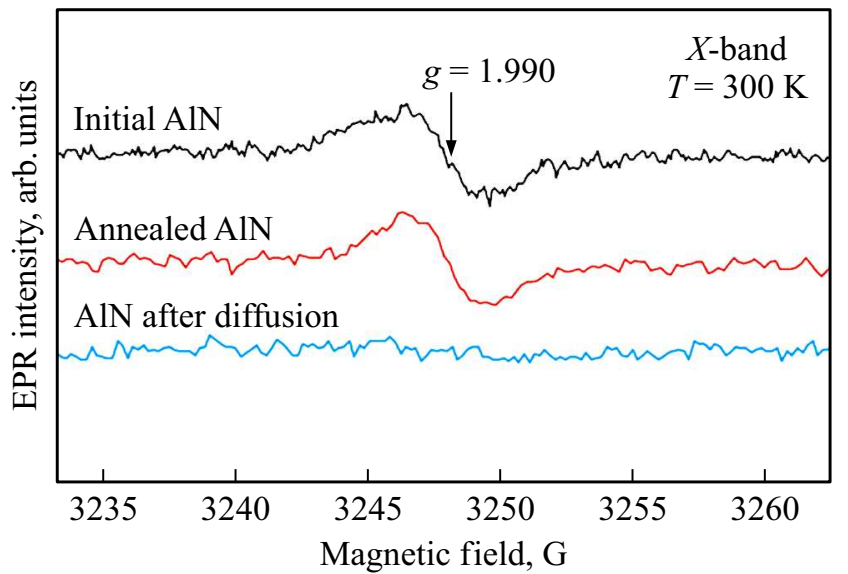

Рис. 1. Спектры ЭПР, зарегистрированные в исходном образце AlN (верхний спектр), после отжига при $T=1880^{\circ} \mathrm{C}$ (средний спектр) и после диффузии Ве (нижний спектр).

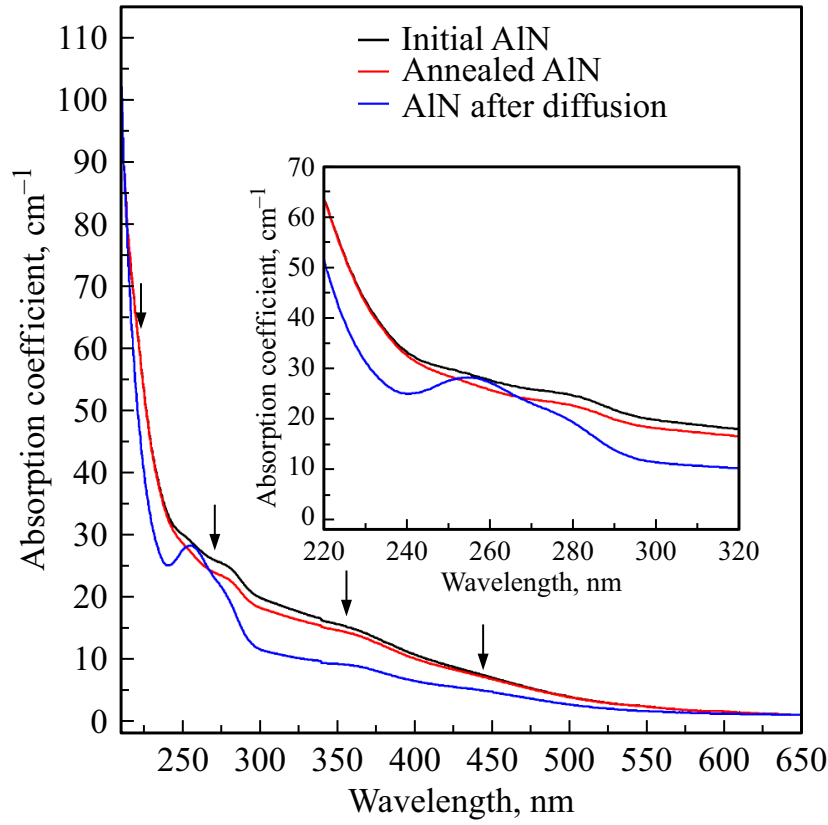

Рис. 2. Оптическое поглощение исходного AlN (initial AlN), после отжига (Annealed AlN) и после диффузии Be (AlN after diffusion). На вставке приведено УФ поглощение в увеличенном масштабе. Стрелками отмечены максимумы полос поглощения.

объяснения данного наблюдения необходимо предположить, что введение примеси Ве, являющейся акцептором в позиции замещения атомов $\mathrm{Al}\left(\mathrm{Be}_{\mathrm{Al}}\right)$ [8], привело к созданию центров акцепторного типа, что вызвало сдвиг уровня Ферми в сторону середины запрещенной зоны, как минимум ниже уровня 0/+ доноров кремния, тем самым приведя к компенсации мелких донорных центров.

Далее нами были исследованы оптические свойства исходного монокристалла $\mathrm{AlN}$, после легирования примесью Ве посредством диффузии, а также после высоко- 
температурного отжига. Результаты этих исследований приведены на рис. 2. Видно, что исходный кристалл $\mathrm{AlN}$ характеризуется незначительным поглощением в видимом диапазоне: полоса поглощения в районе 450 нм ( $\approx 2.75$ эВ), в УФ диапазоне: полосы поглощения на длине волны $360 \mathrm{Hм}(\approx 3.6$ эВ $), 265 \mathrm{Hм}(\approx 4.7$ эВ $)$ и в районе $225 \mathrm{нм}(\approx 5.5$ эВ). Видно, что отжиг не вызвал каких-либо заметных изменений оптического поглощения. Предположительно, наблюдаемые нами полосы оптического поглощения связаны с вакансионными дефектами в решетке AIN и их комплексами, образуемыми с примесными атомами кислорода, который является неконтролируемой примесью. Так, в работе [11] было показано, что поглощение на длине волны $450 \mathrm{Hм}$ $(\approx 2.75$ эВ) может быть объяснено наличием в кристалле вакансий азота $\left(V_{\mathrm{N}}\right)$ в нейтральном зарядовом состоянии. Также это поглощение может быть связано с наличием в кристалле $\mathrm{AlN}$ вакансий $\mathrm{Al}\left(V_{\mathrm{Al}}\right)$, формирующих глубокий уровень $V_{\mathrm{Al}}^{3-/ 2-}$ в запрещенной зоне [12]. Полоса поглощения на длине волны $360 \mathrm{Hм}$ $(\approx 3.4$ эВ), по-видимому, также связана с наличием центров $V_{\mathrm{Al}}^{3-/ 2-}$ [13]. Поглощение на $225 \mathrm{Hм}(\approx 5.5$ эВ $)$ связывают с комплексом $\mathrm{C}_{\mathrm{N}}-\mathrm{Si}_{\mathrm{Al}}$, формирующим глубокий уровень, вблизи потолка валентной зоны [14].

На сегодняшний день существует несколько моделей центров, наличие которых объясняет оптическое поглощение на длине волны 265 нм $(\approx 4.7$ эВ). Так, в работе [15] эта полоса связывается с отрицательно заряженным комплексом, состоящим из трех атомов углерода $\left(\left(\mathrm{C}_{\mathrm{N}}-\mathrm{C}_{\mathrm{Al}}-c-\mathrm{C}_{\mathrm{N}}\right)^{-}\right)$. В работе [14] предлагается объяснение этой полосы оптическим переходом электрона в валентную зону с ионизированной акцепторной примеси углерода в позиции замещения азота $\left(\mathrm{C}_{\mathrm{N}}^{-}\right)$. Наиболее важным, на наш взгляд, считается недавно эмпирически установленный факт того, что интенсивность этой полосы поглощения коррелирует с относительной концентрацией донорных и акцепторных центров. Так, в работе [16] была исследована эволюция этого поглощения в зависимости от концентрации донорной примеси кислорода $\left(\mathrm{O}_{\mathrm{N}}\right.$ - донорная примесь $)$ и углерода $\left(\mathrm{C}_{\mathrm{N}}-\right.$ акцепторная примесь) и было показано, что при концентрациях $\mathrm{O}_{\mathrm{N}}>3 \mathrm{C}_{\mathrm{N}}$ и общей концентрации примесей $\left(\mathrm{O}_{\mathrm{N}}+\mathrm{C}_{\mathrm{N}}\right)$, не превышающей $10^{19} \mathrm{~cm}^{-3}$, поглощение практически отсутствует $\left(\alpha_{265 \text { нм }}<30 \mathrm{~cm}^{-1}\right)$. Наблюдение, сделанное в работе [16], хорошо коррелирует с данными оптического поглощения, выполненными на исходном кристалле AIN. Действительно, из спектров ЭПР следует, что концентрация донорных примесей кремния превышает концентрацию акцепторных примесей, что приводит к низкому коэффициенту $\alpha_{265 \text { нм }}<30 \mathrm{~cm}^{-1}$.

После диффузии Ве спектр ОП значительно изменился. В частности, исчезла полоса поглощения в районе $225 \mathrm{нм}$, и оптическое поглощение в видимой области спектра уменьшилось, оптическое поглощение в районе 265 нм, напротив, возросло. Эти изменения ОП связаны со сдвигом положения уровня Ферми в сторону

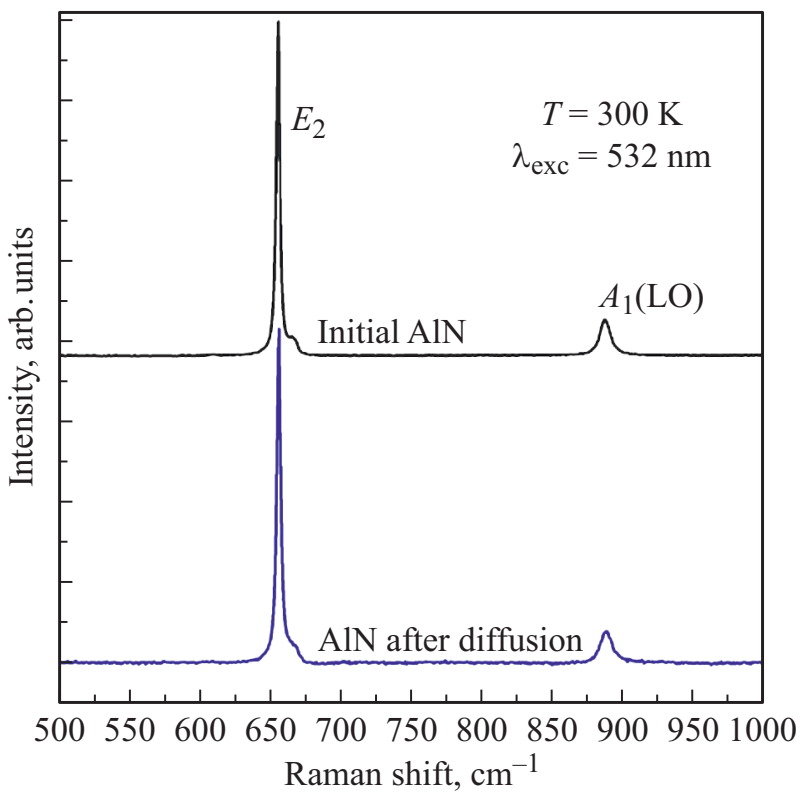

Рис. 3. Спектры КРС, зарегистрированные в кристалле AlN до диффузии (верхний спектр) и после диффузии (нижний спектр).

потолка валентной зоны, что вызывает частичную перезарядку глубоких центров, ответственных за оптическое поглощение как в видимой, так и в УФ областях.

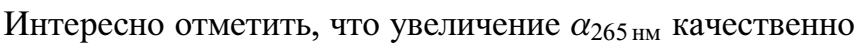
согласуется с данными работы [16]. Действительно, введение акцепторной примеси компенсирует мелкие доноры кремния, тем самым нарушая условие оптической прозрачности в этой полосе $\left(\mathrm{O}_{\mathrm{N}}>3 \mathrm{C}_{\mathrm{N}}\right)$ с той лишь разницей, что вместо доноров кислорода выступают доноры кремния, а вместо акцептороподобной примеси углерода выступают теперь как углерод, так и $\mathrm{Be}_{\mathrm{Al}}$.

Необходимо отметить, что высокотемпературная диффузия Ве не вызвала значительных ухудшений структурного совершенства AlN, что следует из анализа спектров КРС, измеренных до и после диффузии (см. рис. 3). Действительно, положения частот, соответствуюших оптическим фононам $\mathrm{A}_{1}(\mathrm{LO})=890 \mathrm{~cm}^{-1}$ и $E_{2}=657.4 \mathrm{~cm}^{-1}$, находятся в хорошем соответствии с ранее установлеными для AIN высокого структурного совершенства [17].

Известно, что положение линии $E_{2}$ и ее ширина на полувысоте являются мерой внутренних напряжений и дефектности AlN [18]. Из рис. 3 видно, что диффузия Be не привела к сдвигу и уширению линии $E_{2}$.

\section{4. Заключение}

Методом электронного парамагнитного резонанса было показано, что в процессе роста в AlN входит неконтролируемая примесь $\mathrm{Si}$, формирующая мелкий донорный уровень в запрещенной зоне AlN. Установлено, что 
постростовая диффузия Ве приводит к полной компенсации донорной примеси кремния и значительно влияет на оптическое поглощение AlN в UV-C диапазоне.

Таким образом, в работе впервые продемонстрирована эффективная компенсация мелких донорных центров в AlN путем постростового легирования. Получены монокристаллы AlN с низкими значениями показателя оптического поглощения в видимом и УФ диапазонах и высоким структурным соверщенством. Показано, что компенсация донорных центров акцепторной примесью Ве приводит к увеличению оптического поглощения с максимумом на длине волны 265 нм. Данное наблюдение согласуется с ранее установленным фактом, что возникновение данной полосы оптического поглощения определяется относительной концентрацией донорных и акцепторных примесей в $\mathrm{AlN}$ [16]. Методом спектроскопии КРС было показано, что диффузия Ве не вызывает значительного изменения кристаллического совершенства AlN.

\section{Финансирование работы}

Исследование выполнено при частичной финансовой поддержке РФФИ в рамках научного проекта № 19-02-00649.

\section{Конфликт интересов}

Авторы заявляют, что у них нет конфликта интересов.

\section{Список литературы}

[1] J.Y. Tsao, S. Chowdhury, M.A. Hollis, D. Jena, N.M. Johnson, K.A. Jones, R.J. Kaplar, S. Rajan, C.G. Van de Walle, E. Bellotti, C.L. Chua, R. Collazo, M.E. Coltrin, J.A. Cooper, K.R. Evans, S. Graham, T.A. Grotjohn, E.R. Heller, M. Higashiwaki, M.S. Islam, P.W. Juodawlkis, M.A. Khan, A.D. Koehler, J.H. Leach, U.K. Mishra, R.J. Nemanich, R.C.N. Pilawa-Podgurski, J.B. Shealy, Z. Sitar, M.J. Tadjer, A.F. Witulski, M. Wraback, J.A. Simmons. Adv. Electron. Mater., 4, 1600501 (2018).

[2] T.Y. Chemekova, O.V. Avdeev, I.S. Barash, E.N. Mokhov, S.S. Nagalyuk, A.D. Roenkov, A.S. Segal, Y.N. Makarov, M.G. Ramm, S. Davis, G. Huminic, H. Helava. Phys. Status Solidi C, 5, 1612 (2008).

[3] B. Monemar, P.P. Paskov, J.P. Bergman, A.A. Toropov, T.V. Shubina. Phys. Status Solidi B, 244, 1759 (2007).

[4] Y. Taniyasu, M. Kasu, T. Makimoto. Nature, 441, 325 (2006).

[5] S.B. Orlinskii, J. Schmidt, P.G. Baranov, M. Bickermann, B.M. Epelbaum, A. Winnacker. Phys. Rev. Lett., 100, 256404 (2008).

[6] X.Th. Trinh, D. Nilsson, I.G. Ivanov, E. Janzn, A. KakanakovaGeorgieva, N.T. Son. Appl. Phys. Lett., 105, 162106 (2014).

[7] V.A. Soltamov, I.V. Ilyin, A.A. Soltamova, D.O. Tolmachev, N.G. Romanov, A.S. Gurin, V.A. Khramtsov, E.N. Mokhov, Yu.N. Makarov, G.V. Mamin, S.B. Orlinskii. Appl. Magn. Reson., 44, 1139 (2013).
[8] V.A. Soltamov, M.K. Rabchinskii, B.V. Yavkin, O.P. Kazarova, S.S. Nagalyuk, V.Y. Davydov, A.N. Smirnov, V.F. Lebedev, E.N. Mokhov, S.B. Orlinskii, P.G. Baranov. Appl. Phys. Lett., 113, 082104 (2018).

[9] O.V. Avdeev, T.Yu. Chemekova, H. Helava, M.G. Ramm, Yu.N. Makarov, E.N. Mokhov, S.S. Nagalyuk, A.S. Segal. Growth of Bulk AlN Crystals. In: Comprehensive Semiconductor Science and Technology (2011) p. 282. DOI: 10.1016/B978-0-44-453153-7.00094-8/

[10] Ю.П. Маслаковец, Е.Н. Мохов, Ю.А. Водаков, Г.А. Ломакина. ФТТ, 10, 809 (1968). [Yu.P. Maslakovets, E.N. Mokhov, Yu.A. Vodakov, G.A. Lomakina. Sov. Phys. Solid State, 10, 634(1968).].

[11] V.A. Soltamov, I.V. Ilyin, A.A. Soltamova, D.O. Tolmachev, N.G. Romanov, A.S. Gurin, E.N. Mokhov, P.G. Baranov. Phys. Status Solidi C, 9, 745 (2012).

[12] A. Sedhain, L. Du, J.H. Edgar, J.Y. Lin, H.X. Jiang'. Appl. Phys. Lett., 95, 262104 (2009).

[13] Q. Yan, A. Janotti, M. Schefler, C.G. Van de Walle, App. Phys. Lett., 105, 111104 (2014).

[14] B.E. Gaddy, Z. Bryan, I. Bryan, J. Xie, R. Dalmau, B. Moody, Y. Kumagai, T. Nagashima, Y. Kubota, T. Kinoshita, A. Koukitu, R. Kirste, Z. Sitar, R. Collazo, D.L. Irving. Appl. Phys. Lett., 104, 202106 (2014).

[15] K. Irmscher, C. Hartmann, C. Guguschev, M. Pietsch, J. Wollweber, M. Bickermann. J. Appl. Phys., 114, 123505 (2013).

[16] C. Hartmann, J. Wollweber, S. Sintonen, A.A. Dittmar, L. Kirste, S. Kollowa, K. Irmschera, M. Bickermanna. Cryst. Eng. Comm., 18, 3488 (2016).

[17] V.Y. Davydov, Y.E. Kitaev, I.N. Goncharuk, A.N. Smirnov, J. Graul, O. Semchinova, D. Uffmann, M.B. Smirnov, A.P. Mirgorodsky, R.A. Evarestov. Phys. Rev. B, 58, 12899 (1998).

[18] M. Kuball, J.M. Hayes, Y. Shi, J.H. Edgar. Appl. Phys. Lett., 77 (2012).

Редактор А.Н. Смирнов

\section{Effect of Be acceptor impurity on optical properties of AIN single crystals}

\author{
E.N. Mokhov ${ }^{1}$, M.K. Rabchinskiy ${ }^{1}$, S.S. Nagalyuk ${ }^{1}$, \\ M.R. Gafurov ${ }^{2}$, O.P. Kazarova ${ }^{1}$ \\ ${ }^{1}$ loffe Institute, \\ 194021 St. Petersburg, Russia \\ ${ }^{2}$ Kazan (Volga region) Federal University, \\ 420008 Kazan, Russia
}

\begin{abstract}
The effect of high-temperature $\left(T=1880^{\circ} \mathrm{C}\right)$ diffusion of beryllium ions on the properties of single-crystal aluminum nitride is investigated. It was shown that postgrowth doping of AlN with beryllium compensates the small silicon donor centers entering the AlN lattice in an uncontrolled manner during growth. It was established that the introduction of Be into the AlN lattice leads to a decrease in the optical absorption of the latter in the visible and ultraviolet ranges. The totality of the results is explained by a shift in the position of the Fermi level, caused by the introduction of the acceptor impurity of beryllium, towards the ceiling of the AlN valence band.
\end{abstract}

